\title{
Dynamics Behaviours of a Discrete Memristor Map with Fractional Order
}

\author{
Amina Aicha Khennaoui ${ }^{1 *}$ and Adel Ouannas ${ }^{2 \dagger}$ \\ 1 Laboratory of dynamical system and control, University of Larbi Ben M'hidi, Oum El-Bouaghi, Algeria \\ 2 Department of Mathematics and Computer science, University of Larbi Ben M'hidi, Oum El Bouaghi, Algeria
}

\begin{abstract}
In this paper; a simple fractional discrete memristor is designed using discrete fractional calculus, based on this model a fractional order discrete memristor based chaotic map is constructed. Dynamical behaviour of the proposed fractional model is investigated numerically. The impact of fractional order and system parameters on the newly model are illustrated using bifurcation diagrams and phase attractors. It is verified that the newly model has chaos behaviour.

Keywords: Discrete memristor $\bullet$ Discrete fractional calculus • Memristor $\bullet$ Chaos
\end{abstract}

\section{Introduction}

Fractional calculus is a topic which is developed more than 300 years as an extension of differentiation and integration [1]. In comparison with first order derivative, the fractional order ones have benefits in describing the long memory property. By memory effects we mean that the states of the fractional systems is determined by all the previous states. At the same time, during the last decade, attention has been focused on discrete fractional calculus and fractional difference operators [2]. Recently various complex dynamics residing in fractional order iterated map, such as chaos, hyperchaos and coexisting attractors. For example, in [3] the hyperchaotic dynamic of the fractional generalized Hénon map has been investigated. In [4], the chaotic dynamics and combined synchronization of three two dimensional maps have been illustrated; whereas in [5] Almatroud et al. found rich chaotic behaviours of a novel two-dimensional (2D) hyperchaotic fractional map with infinite line of equilibrium. Thanks to this unique characteristics, fractional order iterated map have been deeply studied in academic fields [6-16].

\footnotetext{
* E-mail: kamina_aicha@yahoo.fr

† E-mail: ouannas.adel@univ-oeb.dz
} 
Memristor is the forth basic circuit element which was first proposed by Chua in [17], but it was until 2008 that HP laboratory developed the practical application of it [18]. Till now, memristors have been applied in many fields such as, artificial network and nonlinear circuit [19, 20]. Lately, there has been much work on constructing discrete memristor [21]. For instance, in [22] several two dimensional discrete maps where established by introducing memristor into one dimensional maps. In [23], hyperchaotic phenomena was demonstrated in a discrete memristor coupled logistic map; whereas a 2D non-autonomous discrete memristor-based hyperchaotic map is analyzed [24]. The discrete memristor in comparison with the classical memristor has simplest form and fast numerical computation. Nonetheless, studies on the fractional discrete memristor maps are rare in the literature [25, 26].

The main objective of this article is to investigate the effect of fractional order values on the dynamic behaviour of discrete memristor based map. The dynamical behaviours of the system is numerically analyzed using bifurcation diagrams and phase portraits . The rest of this article is organized as follows. In Section 2, we give some primary preliminaries associated with discrete fractional calculus, and we review the fractional form of discrete memristor. Hence, we introduce a fractional discrete memrister based chaotic map. The dynamics of the proposed fractional discrete memristor map in Section 3 will be discussed in detail, including phase plots and bifurcation diagrams.

\section{Preliminaries and model description}

In order to describe our fractional discrete memristor, some definitions of the discrete fractional calculus are discussed and the mathematical model of the memristor involving the Caputo-left operator is given.

\section{Preliminaries and model description}

In order to describe our fractional discrete memristor, some definitions of the discrete fractional calculus are discussed and the mathematical model of the memristor involving the Caputo-left operator is given.

\subsection{Discrete fractional calculus}

\section{Definition 3.1.}

[27] The fractional sum of order $\gamma$ for a function $g$ on a time scale $N_{a}=\{a, a+1, a+2, \ldots\}$ is defined as

$$
\Delta_{\tau}^{-\gamma} g(\tau)=\frac{1}{\Gamma(\gamma)} \sum_{\tau=a}^{s-\gamma}(s-\tau-1)^{(\gamma-1)} g(\tau)
$$

with $s \in \mathbb{N}_{a+\gamma}, \gamma>0$.

Definition 3.2.

[28] The $\gamma$-Caputo fractional difference operator for a function $g(s)$., is defined as

$$
{ }^{C} \Delta_{a}^{\gamma} g(s)=\Delta_{a}^{-(m-\gamma)} \Delta^{m} g(s)=\frac{1}{\Gamma(m-\gamma)} \sum_{\tau=a}^{s-(m-\gamma)}(s-\tau-1)^{(m-\gamma-1)} \Delta_{\tau}^{m} g(\tau)
$$


where $s \in \mathbb{N}_{a+m-\gamma}, m=\lceil\gamma\rceil+1$ and $\gamma \notin \mathbb{N}$. $(s-\tau-1)^{(m-\gamma-1)}$ and $\Delta_{\tau}^{m} X(\tau)$ are the falling factorial function and the $m$-th integer difference operator, respectively, which are defined as

$$
(s-\tau-1)^{(m-\gamma-1)}=\frac{\Gamma(s-\tau)}{\Gamma(s-\tau-m+\gamma+1)},
$$

and

$$
\Delta^{m} g(s)=\Delta\left(\Delta^{m-1} g(s)\right)=\sum_{k=0}^{m}\left(\begin{array}{c}
m \\
k
\end{array}\right)(-1)^{m-k} g(s+k), \quad s \in \mathbb{N}_{a} .
$$

Now, we shall give a theorem which will allow us to derive the numerical formula of the discrete fractional systems.

Theorem 3.1.

[29] For the fractional difference equation

$$
\left\{\begin{array}{l}
{ }^{C} \Delta_{a}^{\gamma_{i}} x(s)=g\left(s+\gamma_{i}-1, x\left(s+\gamma_{i}-1\right)\right) \\
\Delta^{k} x(s)=x_{k}, \quad m=\left\lceil\gamma_{i}\right\rceil+1, \quad k=0,1, \ldots, m-1
\end{array}\right.
$$

the unique solution of this initial value problem (5) is given by

$$
x(s)=x_{0}(s)+\frac{1}{\Gamma\left(\gamma_{i}\right)} \sum_{\tau=a+m-\gamma_{i}}^{s-\gamma_{i}}(s-\sigma(\tau))^{\left(\gamma_{i}-1\right)} f\left(\tau+\gamma_{i}-1, x\left(\tau+\gamma_{i}-1\right)\right), \quad s \in N_{a+m},
$$

where

$$
x_{0}(s)=\sum_{k=0}^{m-1} \frac{(s-a)^{k}}{\Gamma(k+1)} \Delta_{k} x(a)
$$

\subsection{The fractional discrete memristor}

From the property of circuit theory, memristor is a two terminal device exhibiting a pinched hysteresis for any non-sinusoidal periodic waveform. According to [17], an ideal memristor model is characterized by

$$
\left\{\begin{array}{l}
v(t)=M(q) i(t) \\
\frac{d q}{d t}=i(t)
\end{array}\right.
$$

where $i$ is the current, $v$ is the voltage, $q$ is an inner charge variable and $M(q)$ is given by $M(q)=a+q(t)^{2}+$ $c \sin (\alpha n) i(t)$, in which $a, b, c, \alpha$ are constant. Therefore the mathematical memristor is proposed as

$$
\left\{\begin{array}{l}
v(t)=\left(a q(t)^{2}+b+c \sin (\alpha t)\right) i(t), \\
\frac{d q}{d t}=i(t)
\end{array}\right.
$$

Recently, Deng and Li [24] have defined a discrete memristor by discretizing the continuous memristor model (9) using the forward Euler difference method. The discrete memristor has the following form

$$
\left\{\begin{array}{l}
v(n)=\left(a q(n)^{2}+b+c \sin (\alpha n)\right) i(n), \\
\Delta q(n)=\Delta T i(n)
\end{array}\right.
$$


where $\left.\Delta q(n)=q(n+1)-q_{(} n\right)$ and $\Delta T$ is the iteration step size. According to the numerical simulation reported in [24], the discrete memristor model can exhibit a pinched hysteris loop when a sinusoidal voltage is applied. From the iterated equation $\Delta q_{n}=i(n)$ it is deduced that $q(n)=q_{1}+\sum_{j=1}^{n-1} i(j)$. Hence,

$$
v(n)=\left(a\left(q_{1}+\sum_{j=1}^{n-1} i(j)\right)^{2}+c \sin (\alpha n)\right) i(n) .
$$

From equation (11), it is shows that the discrete memristor has memory effect characteristic similarly to the discrete fractional calculus, so the previous memristor can be extended to fractional order. Based on this equation (11), we changed the first difference operator $\Delta$ with the Caputo like difference operator ${ }^{C} \Delta_{a}^{\gamma}$ and got a new $2 \mathrm{D}$ discrete memristor as

$$
\left\{\begin{array}{l}
v(n)=\left(a q(n)^{2}+b+c \sin (\alpha n)\right) i(n), \\
{ }^{C} \Delta_{a}^{\gamma} q(t)=i(t+1-\gamma)
\end{array}\right.
$$

where $t \in \mathbb{N}_{a+1-\gamma}$. According to Theorem 3.1, the numerical formula of the fractional discrete memristor (12) is designed as

$$
\left\{\begin{array}{l}
v(n)=i(n)\left(a\left(q(0)+\frac{1}{\Gamma\left(\gamma_{1}\right)} \sum_{j=0}^{n-1} \frac{\Gamma(n-1-j+\gamma)}{\Gamma(n-1-j+1)} i(j)\right)^{2}+c \sin (\alpha n)\right) \\
q(n)=q(0)+\frac{1}{\Gamma\left(\gamma_{2}\right)} \sum_{j=0}^{n-1} \frac{\Gamma(n-1-j+\gamma)}{\Gamma(n-1-j+1)} i(j) .
\end{array}\right.
$$

Set the initial condition $q(0)=0$, system parameters $a=1.5, b=-1, c=0.5, \alpha=0.6$, Figure 3.2 shows the relationship curves $v-i$ between the output current and input voltage for different fractional order $\gamma$. This figure is obtained by adding a sin voltage signal $i(n)=A \sin (F n)$, where $A$ is the amplitude and $F$ is the frequency. It visually demonstrate that the relationship curves $v-i$ can display the elegant hysteresis loops pinched at the origin for all the fractional order values.

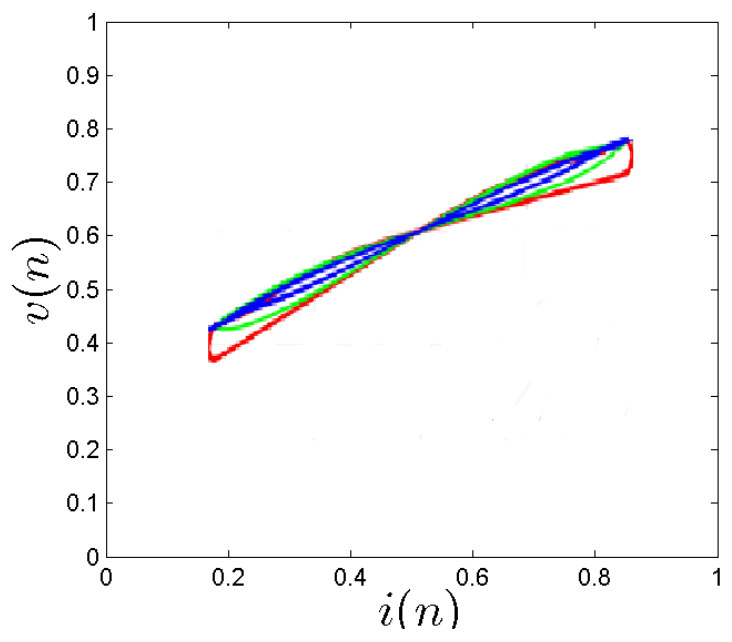

Figure 1. Numerical simulation of the memristors pinched hysteresis 


\subsection{The fractional memristor based $2 \mathrm{D}$ map}

According to reference [24], the mathematical equation of the discrete meristor system is described as

$$
\left\{\begin{array}{l}
y(n+1)=y(n)+\beta_{2} x(n), \\
x(n+1)=\beta_{1}\left(a y(n)^{2}+b+c \sin (\alpha n)\right) x(n) .
\end{array}\right.
$$

In this work, we extend the integer-order discrete memristor system to the fractional-order case. Herein, the first-order difference of the discrete memristor system is formulated as

$$
\left\{\begin{array}{l}
\Delta y(n)=\beta_{2} x(n) \\
\Delta x(n)=\beta_{1}\left(a y(n)^{2}+b+c \sin (\alpha n)\right) x(n) .
\end{array}\right.
$$

Based on this equation (15), we changed the first difference operator $\Delta$ with the Caputo like difference operator ${ }^{C} \Delta_{a}^{\gamma_{i}}$ and got a new $2 \mathrm{D}$ discrete memristor system with fractional order, as follows

$$
\left\{\begin{array}{l}
{ }^{C} \Delta_{a}^{\gamma_{1}} y(t)=\beta_{2} x\left(t-1+\gamma_{1}\right), \\
{ }^{C} \Delta_{a}^{\gamma_{2}} x(t)=\beta_{1}\left(a y\left(t-1+\gamma_{2}\right)^{2}+b+c \sin \left(\alpha\left(t+1-\gamma_{2}\right)\right)\right) x\left(t+1-\gamma_{2}\right) .
\end{array}\right.
$$

for $t \in \mathbb{N}_{a+1-\gamma}$, where $N_{a}=\{a, a+1, a+2, \ldots\}, a \in \mathbb{N}$, and $0<\gamma_{i} \leq 1, i=1,2$ are the fractional orders.

\section{Numerical analysis}

The goal of this section is to explore the chaotic behavior and the complex dynamical characteristics of the fractional discrete memristor system (16) via numerical simulation for the same system parameters given in the above section. In order to illustrate these, we shall first give the numerical formula of the fractional discrete memristor system (16)

$$
\left\{\begin{array}{l}
x(n)=x(0)+\frac{1}{\Gamma\left(\gamma_{1}\right)} \sum_{j=0}^{n-1} \frac{\Gamma\left(n-1-j+\gamma_{1}\right)}{\Gamma(n-1-j+1)} \beta_{2} x(j), \\
y(n)=y(0)+\frac{1}{\Gamma\left(\gamma_{2}\right)} \sum_{j=0}^{n-1} \frac{\Gamma\left(n-1-j+\gamma_{2}\right)}{\Gamma(n-1-j+1)} \beta_{1}\left(a y(j)^{2}+b+c \sin (\alpha(j))\right) x(j),
\end{array}\right.
$$

In the following, the chaotic behavior and the route to chaos of the proposed macroeconomic system with incommensurate fractional order (16) are carefully analyzed via the computation of bifurcation diagrams and phase portraits. In particular, the effects of system parameters, and the fractional order values $\gamma_{i}, \forall i=1,2$ on the dynamics of the model are illustrated in details. All the numerical simulation results are carried on Matlab software. 


\subsection{Bifurcation diagram and phase portrait}

Here, we discuss the dynamics of the fractional discrete memristor (16) for different fractional order values $\gamma_{i}$ when the control parameters are assigned as $a=1.5, b=-1, c=0.04, \alpha=0.5, \beta_{2}=1, \beta_{1}=1.72$ and the initial condition is selected as $(x(0), y(0))=(-0.1,0.1)$.

When the fractional order $\gamma_{1}$ is taken as an adjustable parameter, the bifurcation diagram is shown in Figure 2 , for fractional order $\gamma_{2}=1$. It is easy to observe that there are chaotic region when for any value of $\gamma_{1} \in(0,1]$. To illustrate the dynamics of the fractional discrete system (16) better, phase portraits with different values of $\gamma_{1}$ are presented in Figure 3. From Figure 3, we notice that the proposed fractional discrete memristor show different dynamic chaotic attractors for these corresponding different fractional order values. Moreover, it is concluded that the fractional memristor has complex dynamics with smallest fractional order values. Similarly, setting fractional

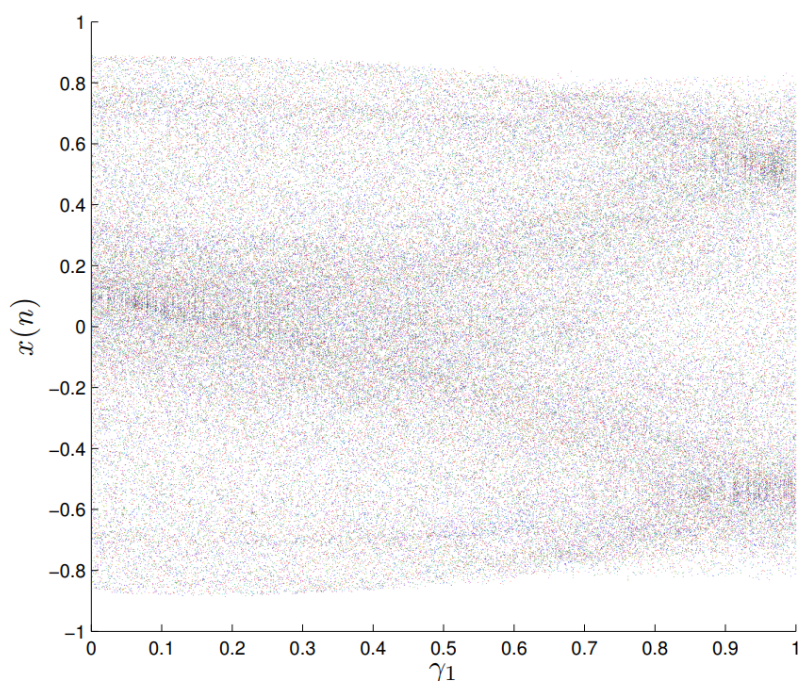

Figure 2. (a) Bifurcation diagram versus $\gamma_{1} \in(0,1]$ of the fractional discrete memristor system (16) with $\gamma_{1}=1$.

order $\gamma_{1}=1$, Figure 4 shows the bifurcation diagram for order $\gamma_{2} \in(0.95,1]$. When $\gamma_{1}$ is used as bifurcation parameter, the fractional discrete memristor system exhibits chaos behaviour. In addition, when the order is less than 0.915 the system will diverge. The dynamic of the fractional discrete system is expressed through the phase portraits as shown in Figure 5. Clearly, the phase portraits in Figure 5 demonstrate the changes in the complex dynamical behaviours of the fractional discrete memristor map. The above numerical simulation show that the dynamic behaviors of the fractional mamristor system (16) are distinctly different when the fractional orders are incommensurate from those in which the integer order. 

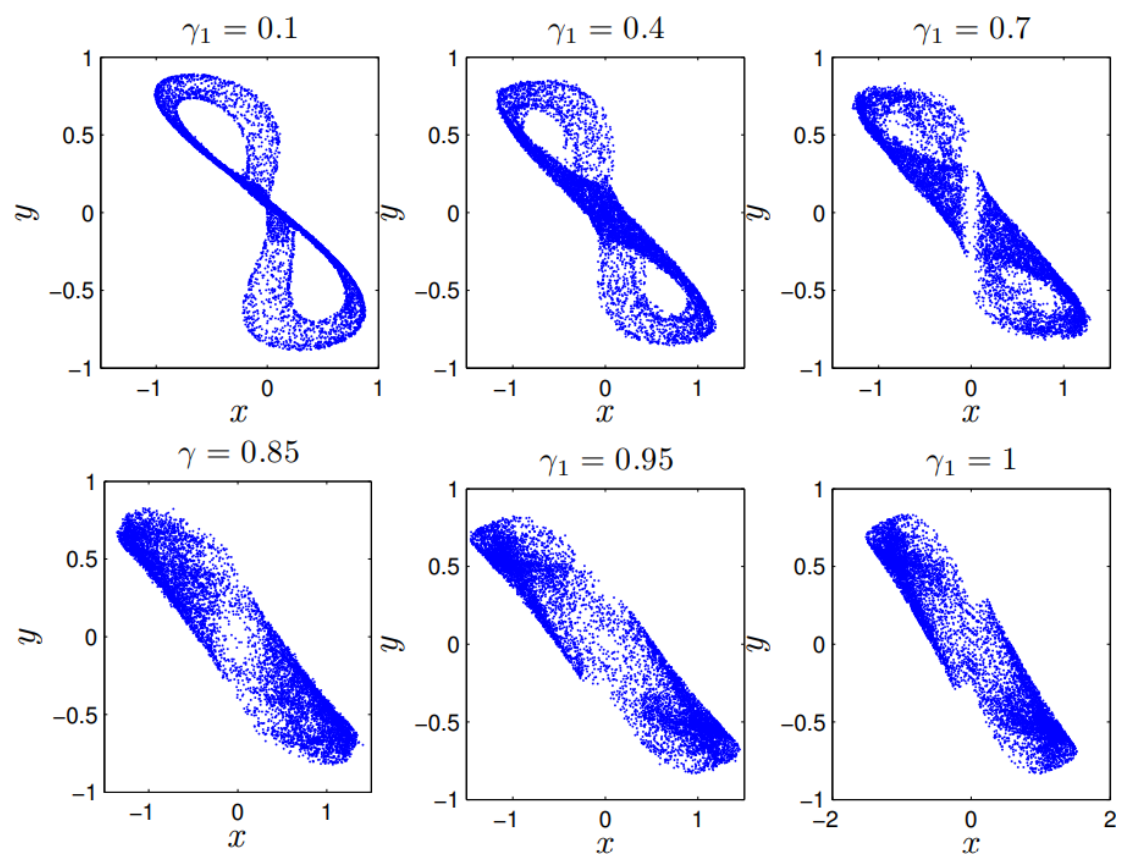

Figure 3. (a) Different phase portraits of the fractional memristor discrete system (16) for different fractional order values.

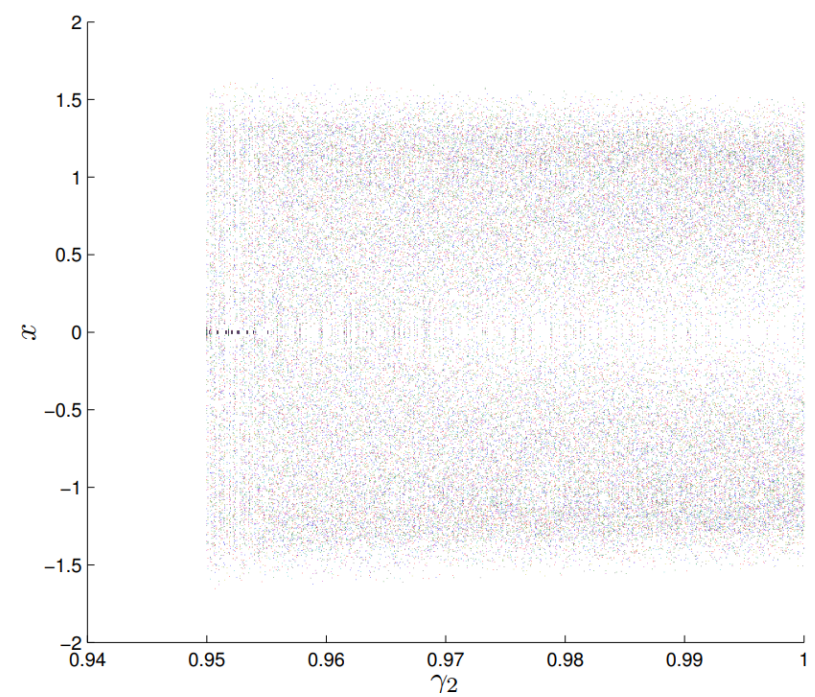

Figure 4. (a) Bifurcation diagram versus $\gamma_{2} \in(0.95,1]$ of the fractional discrete memristor system (16) with $\gamma_{2}=1$.

\section{Conclusions}

This paper has considered a new 2D fractional discrete memristor map based on the Caputo-type delta difference operator. Through phase portraits and bifurcation diagrams, the complex dynamics of this proposed map, with some changes in the incommensurate fractional-order, are discussed. Results have shown that chaos exists in this fractional-order system and that the type and range of chaotic behavior depend on the fractional order values. 

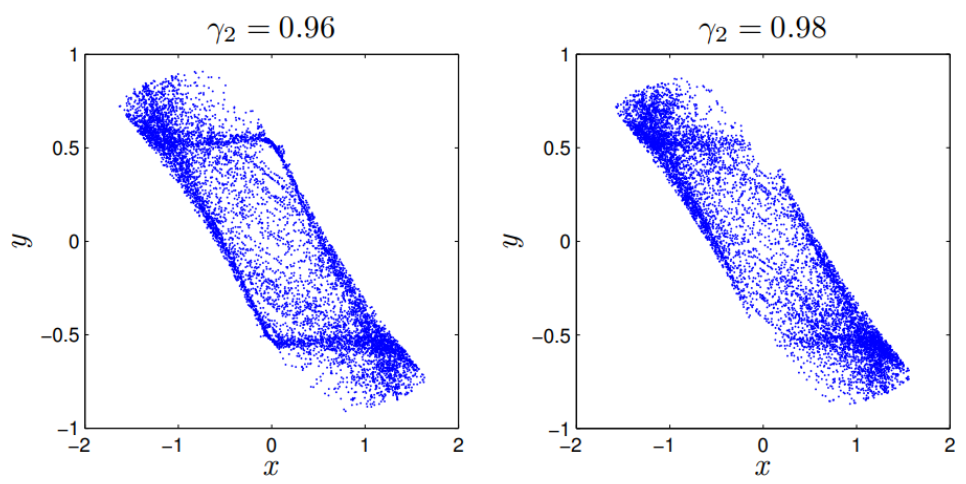

Figure 5. Different phase portraits of the fractional memristor discrete system (16) for different fractional order values $\gamma_{2}$.

Compared with the integer order discrete memristor, the fractional discrete-time one has more complexity when fractional order values $\left(\gamma_{i}, i=1,2,3\right)$ smaller. Due to the rich complex dynamical behaviour, this research can provide theoretical basis and help for the research in electronic circuit.

\section{Funding}

This research received no external funding.

\section{Competing Interests}

The authors declare that there are no conflicts of interest regarding the publication of this paper.

\section{References}

[1] P. Ostalczyk, Discrete fractional calculus: applications in control and image processing, World scientific, 2015 .

[2] M. Edelman, E. E. Macau, M. A. Sanjuan, Chaotic, fractional, and complex dynamics: new insights and perspectives. Springer International Publishing, 2018.

[3] M. K. Shukla, B. B. Sharma, Investigation of Chaos in Fractional Order Generalized Hyperchaotic Hénon Map. AEU-International Journal of Electronics and Communications. 78 (2017), 265-273.

[4] A. A. Khennaoui, A. Ouannas, S. Bendoukha, G. Grassi, R. P. Lozi, V. T. Pham, On fractional-order discrete-time systems: Chaos, stabilization and synchronization, Chaos, Solitons \& Fractals, 119 (2019), 150-162.

[5] A. O. Almatroud, A. A. Khennaoui, A. Ouannas, V. T. Pham, Infinite line of Equilibriums in a novel fractional map with coexisting infinitely many attractors and initial offset boosting, International Journal of Nonlinear Science and Numerical Simulation, 2021. 
[6] A. Ouannas, A. A. Khennaoui, S. Bendoukha, G. Grassi, On the Dynamics and Control of a Fractional Form of the Discrete Double Scroll, International. Journal of Bifurcation and Chaos. 29 (2019), 1950078.

[7] A. Ouannas, A. A .Khennaoui, G. Grassi, S. Bendoukha, On chaos in the fractional-order Grassi-Miller map and its control, Journal of Computational and Applied Mathematics. 358 (2019), 293-305.

[8] L. Jouini, A. Ouannas, A. A. Khennaoui, X. Wang, G. Grassi, V. T. Pham, The fractional form of a new three-dimensional generalized Hénon map, Advances in Difference Equations. 122 (2019).

[9] A. Ouannas, A. A. Khennaoui, Z. Odibat, V. T. Pham, G. Grassi, On the dynamics, control and synchronization of fractional-order Ikeda map, Chaos, Solitons 6 Fractals. 123 (2019), 108-115.

[10] A. Ouannas, A. A. Khennaoui, S. Bendoukha, Z . Wang, V. T. Pham, The Dynamics and Control of the Fractional Forms of Some Rational Chaotic Maps, Journal of Systems Science and Complexity. (2020), 1-20.

[11] A. Gasri, A. Ouannas, A. A. Khennaoui, S. Bendoukha, V. T. Pham, On the Dynamics and Control of Fractional Chaotic Maps with Sine Terms, International Journal of Nonlinear Sciences and Numerical Simulation. 1(2020).

[12] A. A. Khennaoui, A. Ouannas, S. Boulaaras, V. T. Pham, A. Taher Azar, A fractional map with hidden attractors: chaos and control, The European Physical Journal Special Topics. 229 (2020), 1083-1093.

[13] A. Ouannas, A. A. Khennaoui, S. Momani, G. Grassi, V. T. Pham, Chaos and control of a three-dimensional fractional order discrete-time system with no equilibrium and its synchronization, AIP Advances. 10 (2020), 045310.

[14] A. Ouannas, A. A. Khennaoui, S. Momani, V. T. Pham, R. El-Khazali, Hidden attractors in a new fractionalorder discrete system: Chaos, complexity, entropy and control, Chinese Physics B 1 (2020).

[15] A. Ouannas, A. A. Khennaoui, S. Momani, G. Grassi, V. T. Pham, R. El-Khazali, A Quadratic Fractional Map without Equilibria: Bifurcation, 0-1 Test, Complexity, Entropy, and Control, Electronics. 9 (2020), 748.

[16] A. Ouannas, O. A. Almatroud, A. A. Khennaoui, M. M. Al-sawalha, D. Baleanu, Bifurcations, Hidden Chaos and Control in Fractional Maps, Symmetry. 12 (2020), 879.

[17] L. O. Chua, Memristor-The missing circuit element, IEEE Trans Circuit Theory, 18 (1971), 507-519.

[18] D. B. Strukov, G. S. Snider, D. R. Steward, The missing memristor found. Nature. 453 (2008), 80-83.

[19] T. Raja, S. Mourad, Digital logic implementation in memristor-based crossbars-a tutorial, Fifth IEEE International Symposiuom on IEEE, (2010), 303-309.

[20] M. Teimoory, A. Amirsoleimami, J. Shamsi, Optimized implementation of memristor-based full adder by material implication logic. 2014, 21st IEEE conference on IEEE 2015. 85-92.

[21] Y. Peng, S. He, K. Sun, Chaos in the discrete memristor-based system with fractional-order difference. Results in Physics, 24 (2021), 104106.

[22] H. Bao, Z. Hua, H. Li, M. Chen, B. Bao, Discrete Memristor Hyperchaotic Maps. IEEE Transactions on Circuit and systems I. 2021. 
[23] B. Bao, K. Rong, H. Li, K. Li, Z. Hua, X. Zhang. Memristor Coupled Logistic Hyperchaotic map. IEEE Transactions on Circuits and systems II. 2021.

[24] Y. Deng, Y. Li, Bifurcation and bursting oscillations in 2D non-autonomous discrete memristor-based hyperchaotic map. Chaos, Solitons \& Fractals, 150 (2021), 111064.

[25] B. Yan, S. He, S. Wang. Multistability and Formation of Spiral Waves in a Fractional-Order MemristorBased Hyperchaotic Lu System with No Equilibrium Points. Mathematical Problems in Engineering, 12 (2020), 2468134

[26] Y. Peng, S. He, K. Sun, Chaos in the discrete memsristor based system with fractional order difference. Results in Physics, 24 (2021), 104106.

[27] F. M. Atici, P. Eloe, Discrete fractional calculus with the nabla operator. Electronic Journal of Qualitative Theory of Differential Equations, (2009), 1-12.

[28] T. Abdeljawad, On Riemann and Caputo fractional differences, Computers \& Mathematics with Applications, 62(2011), 1602-11.

[29] G. C. Wu, D. Baleanu, Discrete fractional logistic map and its chaos. Nonlinear Dynamics, 75 (2014), 283-287. 